Article

\title{
Preparation and Characterization of Carbamazepine Cocrystal in Polymer Solution
}

\author{
Hao Zhang ${ }^{1}$, Ying Zhu ${ }^{1}$, Ning Qiao ${ }^{1,2, *} \mathbb{1}$, Yang Chen ${ }^{1,2}$ and Linghuan Gao ${ }^{3}$ \\ 1 College of Materials Science and Engineering, North China University of Science and Technology, \\ Tangshan 063210, China; zngzhang720@163.com (H.Z.); YingZhu20171003@163.com (Y.Z.); \\ chenyang@ncst.edu.cn (Y.C.) \\ 2 Hebei Provincial Key Lab of Inorganic Nonmetallic Materials, College of Materials Science and Engineering, \\ North China University of Science and Technology, Tangshan 063210, China \\ 3 College of Basic Medical Sciences, North China University of Science and Technology, Tangshan 063210, \\ China; gaolinghuan@ncst.edu.cn \\ * Correspondence: qiaoning986@163.com; Tel.: +86-315-880-5620
}

Received: 1 October 2017; Accepted: 29 November 2017; Published: 1 December 2017

\begin{abstract}
In this study, we attempted to prepare carbamazepine (CBZ) cocrystal through the solution method in ethanol-water solvent mixture (volume ratio 1:1) and polyvinyl pyrrolidone (PVP) solution. Nicotinamide (NIC) and saccharin (SAC) were selected as cocrystal coformers. Cocrystal screening products were characterized by Fourier Transform Infrared Spectroscopy (FTIR), Differential Scanning Calorimetry (DSC), and Powder X-ray Diffraction (PXRD) techniques. Characterization results show that in ethanol-water solvent mixture, pure CBZ-NIC cocrystal can be prepared, while CBZ-SAC cocrystal cannot be obtained. The addition of PVP can inhibit CBZ-NIC cocrystal formation and facilitate CBZ-SAC cocrystal formation.
\end{abstract}

Keywords: cocrystal preparation; polyvinyl pyrrolidone; carbamazepine; nicotinamide; saccharin

\section{Introduction}

Pharmaceutical cocrystal is a crystalline material with two components present in definite stoichimetric amounts [1]. One of the pharmaceutical cocrystal components is an active pharmaceutical ingredient (API), and the other component is a cocrystal coformer (CCF). Pharmaceutical cocrystal is designed and prepared based on the supramolecular chemistry and crystal engineering theory. API and CCF are combined to form cocrystal through intermolecular interactions such as hydrogen bond (H-bond), $\pi-\pi$ stacking force, van der Waals contact forces [2-4]. Recently, pharmaceutical cocrystal has been demonstrated to be a useful approach to modify physicochemical properties of drugs [5,6]. Among all the physicochemical properties, solubility and dissolution behavior are important properties which take great role in bioavailability of drugs [7]. A pharmaceutical cocrystal can be prepared with the intention to improve solubility behavior of an API without affecting its intrinsic molecular structure. For biopharmaceutics classification system class II (BCS II) drugs, which have high permeability and low solubility, pharmaceutical cocrystal has becoming a popular method to improve its solubility [8]. Carbamazepine (CBZ, IUPAC name 5H-dibenz $(b, f)$ azepine-5-carboxamide, CBZ) is a typical BCS II drug whose bioavailability is limited by its relative low solubility. A high dose is usually administered to achieve the expected treatment effect. During the last decade, CBZ cocrystal has been studied to improve its solubility behavior [9-14]. Two main methods for cocrystal preparation are the grinding method and the solution method. It is difficult to obtain cocrystal product with high purity and good quality using the grinding method [15-17]. The solution method has been applied in many cases to prepare cocrystal $[18,19]$. In the solution method, the selection of solvent is very important. The ideal solvent for the solution cocrystallization process is one in which API and CCF 
has similar molar solubility. Poorly water soluble APIs, such as CBZ, API, and CCF, usually have unequivalent molar solubility in water. The cocrystallization through evaporation of an equimolar solution may result in crystallizing a single cocrystal component or a mixture of individual component and cocrystal. In order to solve this problem, the reaction cocrystallisation (RC) approach has been adopted. In the RC experiment process, the less soluble cocrystal component should be added into saturated or nearly saturated solutions of the other cocrystal component. When the solution becomes supersaturated with respect to cocrystal, the cocrystal product will gradually precipitate. Compared with the evaporation of an equimolar solution method, the experimental operation of RC method is relatively more complex $[20,21]$. Another method is cocrystallization with solvent mixture. In this method, a solvent mixture is used for the solution-mediated cocrystallization process. Solvent mixtures can reduce the solubility differences between cocrystal components; facilitate nucleation rate and provide improved transformation kinetics for cocrystallization [22-24]. Di Profio has successfully prepared carbamazepine-saccharin cocrystal in water/ethanol solvent mixtures by membrane-based crystallization technology [25]. In this study, ethanol-water solvent mixture was also applied to try to prepare CBZ cocrystal through a combination of solution evaporation and the cooling crystallization method. Meanwhile, a polymer additive was added in the solution to investigate the effect of polymer on pharmaceutical cocrystal formation. Previous research studied the effects of polymers on cocrystal stability and dissolution process. In some case, selected polymers can maintain structure stability of certain cocrystals and inhibit the precipitation of individual cocrystal components during the dissolution process [26-29]. From the point of view of dissolution equilibrium, cocrystal preparation via the solution method and cocrystal dissolution can be seen as the two directions of the dissolution equilibrium. Considering that polymers could impart structure stability to cocrystal during its dissolution in some cases, it is expected that the addition of polymer may help cocrystal formation through solution method. In this paper, the effects of polymer on cocrystal preparation process was studied. CBZ was selected as model drug, nicotinamide (NIC, IUPAC name pyridine-3-carboxamide) and saccharin (SAC, IUPAC name 1,1-dioxo-1,2-benzothiazol-3-one) as CCF, and polyvinyl pyrrolidone (PVP) as the representative polymer. The molecular structure of CBZ, NIC, SAC and PVP are shown in Figure 1. PVP is widely used in pharmacy industry as excipient [27,30-34]. In the macromolecule structure of PVP, it has both a hydrophilic and hydrophobic group. It is highly soluble in water and ethanol. During the cocrystallization process, PVP will not precipitate from the solution due to its high solubility and low amount of addition. It can stay in solution as its original dissolved state.

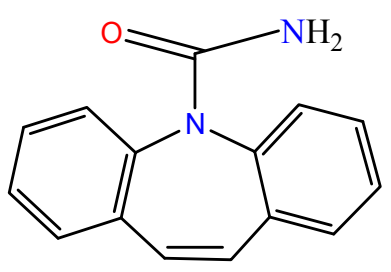

Carbamazepine<smiles>O=C1NS(=O)(=O)c2ccccc21</smiles>

Saccharin<smiles>NC(=O)c1cccnc1</smiles>

Nicotinamide<smiles>CC(C)CC(C)N1CCCC1=O</smiles>

Polyvinyl pyrrolidone

Figure 1. Molecular structure of carbamazepine, nicotinamide, saccharin, and polyvinyl pyrrolidone. 
In this study, we attempted to prepare 1:1 molecular ratio cocrystal of CBZ-NIC and CBZ-SAC in 1:1 volume ratio of ethanol-water solvent mixture with and without PVP. Cocrystal screening products were characterized by Fourier Transform Infrared Spectroscopy (FTIR), Differential Scanning Calorimetry (DSC), and Powder X-ray Diffraction (PXRD). The effects of PVP on CBZ cocrystal formation process have been fully discussed.

\section{Materials and Methods}

\subsection{Materials}

CBZ (>99\% purity) was purchased from Jianglai Reagent Co., Ltd. (Shanghai, China) and used as received. Nicotinamide (NIC, $\geq 99 \%$ purity) and saccharin (SAC, $\geq 98 \%$ purity) were obtained from Aladdin Industrial Co. (Shanghai, China) and used as received. Polyvinylpyrrolidone K30 (PVP) purchased from Tianjin Guangfu Fine Chemical Research Institute (Tianjin, China) was used as received. Ethanol from Yongda Chemical Reagent Co., Ltd. (Tianjin, China) was analytical grade and used as received. Potassium bromide ( $\mathrm{KBr}, \geq 99 \%$ purity) purchased from Jinke Chemical Reagent Co., Ltd. (Tianjin, China) was dried for $24 \mathrm{~h}$ at $50{ }^{\circ} \mathrm{C}$ and finely grounded just before use.

\subsection{Sample Preparation}

\subsubsection{Preparation of CBZ-NIC, CBZ-SAC Cocrystal in Ethanol-Water Solvent Mixture}

A mixture of CBZ (472.6 mg, $2 \mathrm{mmol}$ ) and NIC (244.3 mg, $2 \mathrm{mmol}$ ) (or SAC, $366.3 \mathrm{mg}, 2 \mathrm{mmol}$ ) was added in $20 \mathrm{~mL}$ ethanol-water solvent mixture (with volume ratio of 1:1) in a $50 \mathrm{~mL}$ crystallizer flask. The flask was sealed and stirred under $50{ }^{\circ} \mathrm{C}$ for $20-50 \mathrm{~min}$. After complete dissolution, the stirring and heating of the flask was stopped. The clear solution was cooled down slowly to room temperature. During the cooling process, a small amount of fine crystal was precipitated in the bottom of the flask. Then the solution was placed in a fume hood and allowed to evaporate slowly. After $24 \mathrm{~h}$, about two fifths of the solvent has been evaporated, and more solid product precipitated. The solution was filtered and the solid residue was collected and dried for $12 \mathrm{~h}$ under vacuum. CBZ-NIC cocrystal and CBZ-SAC cocrystal screening product prepared in ethanol-water solvent mixture were obtained through the above procedures. The two cocrystal screening products were abbreviated as CBZ-NIC-E/W and CBZ-SAC-E/W, respectively.

\subsubsection{Preparation of CBZ-NIC, CBZ-SAC Cocrystal in PVP Solution}

The PVP solution was prepared by dissolving $80 \mathrm{mg}$ PVP into $20 \mathrm{~mL}$ ethanol-water solvent mixture. We tried to prepare the CBZ-NIC cocrystal and CBZ-SAC cocrystal in the PVP solution through the same procedures described in Section 2.2.1. The two cocrystal screening products were abbreviated as CBZ-NIC-PVP and CBZ-SAC-PVP respectively.

\subsection{Methods}

\subsubsection{Solubility Test}

The solubility of CBZ, NIC, and SAC in all solvents used in this study (water, ethanol, and ethanol-water mixture with and without PVP) was tested at room temperature, respectively. Excess amount of solid samples were added into $10 \mathrm{~mL}$ solvent and vortexes for $20 \mathrm{~s}$. The suspension was placed in horizontal air-shaking bath at $100 \mathrm{rpm}$ for $24 \mathrm{~h}$. Aliquots were filtered and diluted properly for determination of the concentrations of tested samples by Lambda 750S UV/Vis/NIR Spectrometer (PerkinElmer, Waltham, MA, USA). Concentration of CBZ, NIC, and SAC has been measured under the wavelength of 285,260 , and $276 \mathrm{~nm}$ respectively. The experiments were carried out in triplicate. 


\subsubsection{Fourier Transform Infrared Spectroscopy (FTIR)}

IR spectra of all CBZ cocrystal screening product were characterized by a Bruker VERTEX 70 FTIR spectrometer (Bruker Corporation, Karlsruhe, Germany). The spectrometer resolution is $2 \mathrm{~cm}^{-1}$; spectra recorded from 4000 to $400 \mathrm{~cm}^{-1}$ and number of runs per spectrum 5 . The tested sample was processed by compressing the mixture of sample and $\mathrm{KBr}$ into a thin disk (sample concentration $2 \mathrm{mg}$ in $20 \mathrm{mg} \mathrm{KBr}$ ).

\subsubsection{Differential Scanning Calorimetry (DSC)}

Thermal analyses of all CBZ cocrystal screening product were tested by a NETZSCH DSC 200F3 (NETZSCH Group, Selb, Germany). About $10 \mathrm{mg}$ of tested samples were put in aluminum pans (NETZSCH, JYL0040 $\Phi 8 \times 2.1 \mathrm{~mm}$ ) and covered with a pierced lid. Measurements were carried out at a heating rate of $10{ }^{\circ} \mathrm{C} / \mathrm{min}$ in the temperature range of 20 to $300{ }^{\circ} \mathrm{C}$ under a nitrogen flow rate of $20 \mathrm{~mL} / \mathrm{min}$.

\subsubsection{Powder X-ray Diffraction (PXRD)}

PXRD analysis was performed by Rigaku D/max 2500 PC diffractometer (Rigaku Corporation, Tokyo, Japan). Instrument settings were: monochromatic $\mathrm{Cu} / \mathrm{K} \alpha$ radiation $(\lambda=1.54180 \AA)$, $40 \mathrm{kV} / 100 \mathrm{~mA}, 2 \theta$ region $3-40^{\circ}$, step of $0.02^{\circ}$ and scan rate of $10^{\circ} / \mathrm{min}$.

\section{Results and Discussion}

\subsection{Solubility Test}

The solubility of CBZ, NIC, and SAC in water, ethanol, and ethanol-water mixture with and without PVP was tested at room temperature. The concentration of samples in each solvent was measured by UV-VIS spectrometer and calculated based on corresponding calibration curve. Solubility data was shown in Table 1 . CBZ is poorly soluble in water with a solubility of 0.00001 (mol fraction), while NIC has a relative high solubility in water. The solubility difference between NIC and CBZ has been significantly reduced from 0.09242:0.00001 in water to 0.01961:0.00011 in ethanol-water mixture. The solubility difference between SAC and CBZ has also been decreased in ethanol-water mixture. Solvent mixture and PVP have differential solubilization ability for different compounds. The addition of PVP can increase solubility of all compounds, and the solubility difference between NIC and CBZ has also been slightly increased compared with that in ethanol-water.

Table 1. Solubility of carbamazepine (CBZ), Nicotinamide (NIC), and saccharin (SAC) in different solvents (mol fraction); PVP (polyvinyl pyrrolidone).

\begin{tabular}{ccccc}
\hline API/CCF & Water & Ethanol & Ethanol-Water & Ethanol-Water + PVP \\
\hline CBZ & 0.00001 & 0.00500 & 0.00011 & 0.00014 \\
NIC & 0.09242 & 0.17656 & 0.01961 & 0.02963 \\
SAC & 0.00032 & 0.00931 & 0.00127 & 0.00141 \\
\hline
\end{tabular}

\subsection{FTIR Analysis}

The IR spectra for starting material of CBZ, NIC, and CBZ-NIC cocrystal screening products are shown in Figure 2. IR spectrum for CBZ has two characteristic peaks at 3465, $1675 \mathrm{~cm}^{-1}$, which correspond to the stretching of amine $\left(\mathrm{NH}_{2}\right)$ and carbonyl $(\mathrm{C}=\mathrm{O})$ group of $\mathrm{CBZ}$. The IR spectrum for NIC has a characteristic peak at $3370 \mathrm{~cm}^{-1}$ for the stretching of amine group and double characteristic peaks at 1705 and $1683 \mathrm{~cm}^{-1}$ for the stretching of carbonyl group. The IR spectrum of cocrystal screening product CBZ-NIC-E/W is different from that of the starting material of CBZ or NIC. The characteristic peak of CBZ in CBZ-NIC-E/W has red shift from 3465 and $1675 \mathrm{~cm}^{-1}$ to 3450 and $1660 \mathrm{~cm}^{-1}$, respectively. The characteristic peak of NIC in CBZ-NIC-E/W has a blue shift to 3393 
and $1687 \mathrm{~cm}^{-1}$. The characteristic peak shift can be explained by the formation of $\mathrm{H}$-bond, and the IR spectrum features of sample CBZ-NIC-E/W are in agreement with that of CBZ-NIC cocrystal $[9,35]$. IR characterization results can preliminarily demonstrate the formation of CBZ-NIC cocrystal in ethanol-water solvent mixture. For sample CBZ-NIC-PVP, its IR spectrum has shown some difference from that of CBZ and NIC. The CBZ characteristic peak has shifted to 3450, $1663 \mathrm{~cm}^{-1}$. However, an obvious peak at $1683 \mathrm{~cm}^{-1}$ corresponding for NIC can still be observed, which demonstrated the existence of NIC. IR results preliminarily demonstrate that the possibility of formation of pure CBZ-NIC cocrystal in PVP solutions has been eliminated.

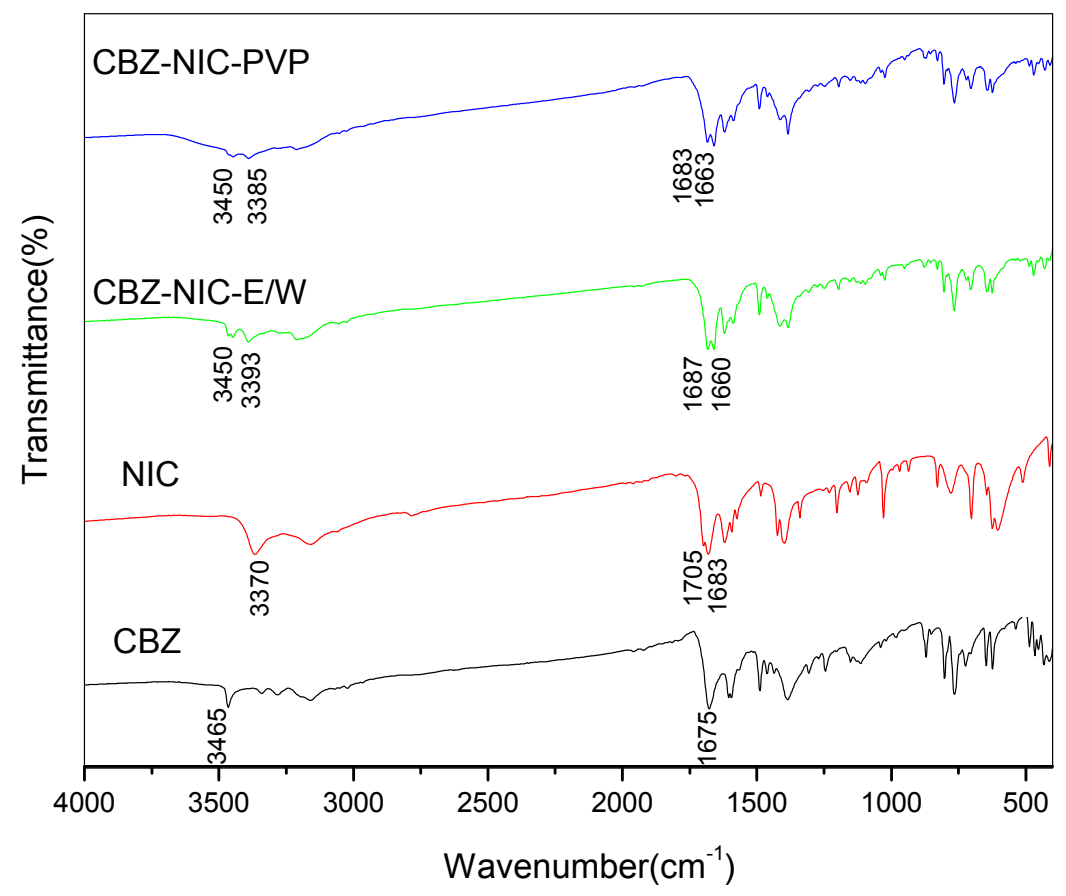

Figure 2. Infrared spectroscopy (IR) spectra of carbamazepine (CBZ), Nicotinamide (NIC), CBZ-NIC cocrystal screening product prepared in ethanol-water mixture (CBZ-NIC-E/W) and in polyvinyl pyrrolidone solution (CBZ-NIC-PVP).

The IR spectra for CBZ, SAC, and CBZ-SAC cocrystal screening products are shown in Figure 3. SAC has characteristic peaks at $3094,1713 \mathrm{~cm}^{-1}$ corresponding for stretch of amine and carbonyl group; peaks at $1338,1180 \mathrm{~cm}^{-1}$ for the asymmetrical and symmetrical stretch of $\mathrm{SO}_{2}$ group. IR spectrum of sample CBZ-SAC-E/W has characteristic peaks for both CBZ and SAC, which demonstrate this sample is a physical mixture of CBZ and SAC. IR spectrum of sample CBZ-SAC-PVP is difference from that of single component of CBZ or SAC, which has shown characteristic peaks shift to 3497, $1648 \mathrm{~cm}^{-1}$ for CBZ, and peak at $1726 \mathrm{~cm}^{-1}$ for SAC. The features of sample CBZ-SAC-PVP is the same as that of CBZ-SAC cocrystal [11]. To conclude, CBZ-SAC cocrystal can be prepared in PVP solutions. 


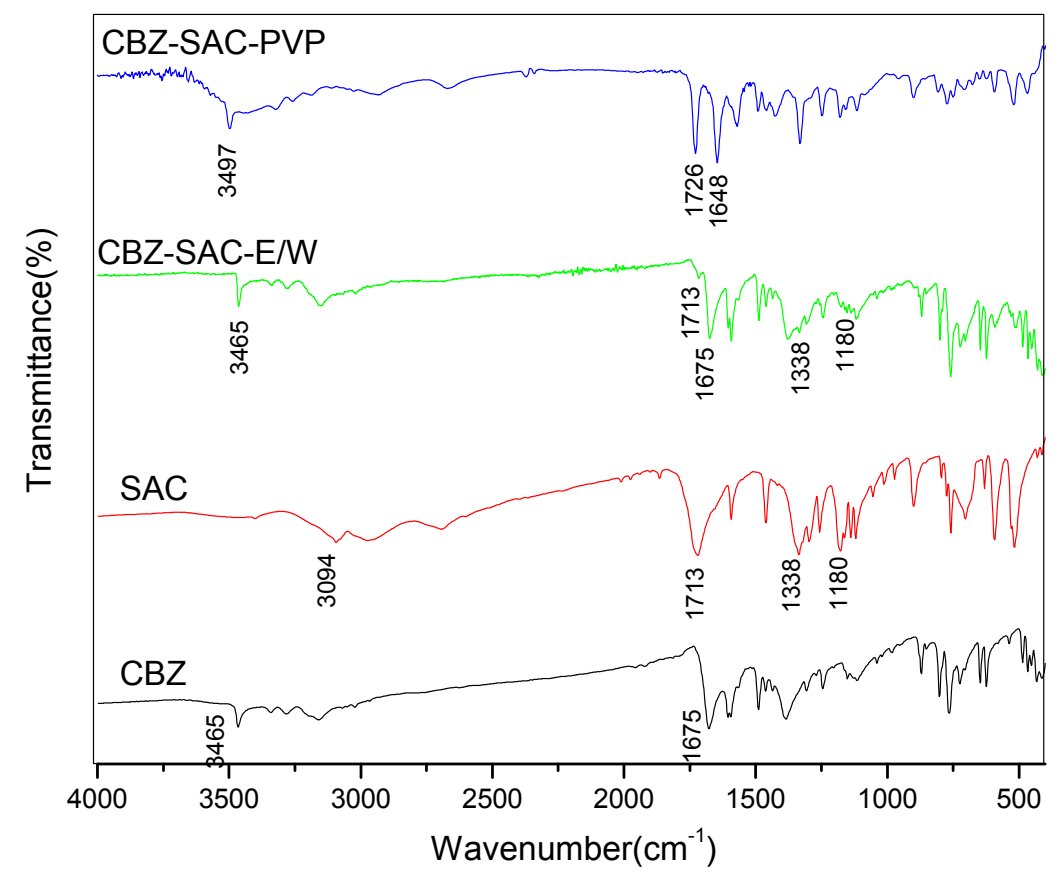

Figure 3. IR spectra of carbamazepine (CBZ), saccharin (SAC), CBZ-SAC cocrystal screening product prepared in ethanol-water mixture (CBZ-SAC-E/W) and in polyvinyl pyrrolidone solution (CBZ-SAC-PVP).

\subsection{DSC Analysis}

DSC curves of CBZ, NIC, CBZ-NIC-E/W, and CBZ-NIC-PVP were shown in Figure 4. As shown in Figure 4, DSC curve of starting material of CBZ and NIC have an endothermic peak attributed to melting around $195{ }^{\circ} \mathrm{C}$ and $132{ }^{\circ} \mathrm{C}$, respectively. Sample CBZ-NIC-E/W has a single endothermal peak, which illustrates that this sample is a pure product with only one molecule structure. The single endothermal peak is around $162{ }^{\circ} \mathrm{C}$, which is located between the melting point of NIC and CBZ. This peak should be assigned to the melting of CBZ-NIC cocrystal [9]. DSC thermal analysis further demonstrated the formation of pure CBZ-NIC cocrystal in an ethanol-water solvent mixture. Sample CBZ-NIC-PVP has two major thermal peaks around 124 and $157^{\circ} \mathrm{C}$ in its DSC curve. The first thermal event around $124{ }^{\circ} \mathrm{C}$ could be due to the melting of NIC and followed by cocrystallization [14]. The second thermal event around $157^{\circ} \mathrm{C}$ could be due to the melting of CBZ-NIC cocrystal, which is formed during the heating process [13]. Compared with pure NIC and cocrystal, the melting point of the two substances in the mixture both reduced. This could be explained by the existence of the second substance.

DSC curves of CBZ, SAC, CBZ-SAC-E/W, and CBZ-SAC-PVP were shown in Figure 5. For sample CBZ-SAC-E/W, the two overlaid peaks of 176 and $181^{\circ} \mathrm{C}$ indicate that this sample is a physical mixture. The two starting materials in the mixture melt one after another in a broad melting range at relative lower temperature compared to their pure state. Sample CBZ-SAC-PVP shows a single endothermal peak at $173^{\circ} \mathrm{C}$, corresponding to the melting point of CBZ-NIC cocrystal [36]. DSC analyses further demonstrate the formation of pure CBZ-SAC cocrystal in PVP solution. 


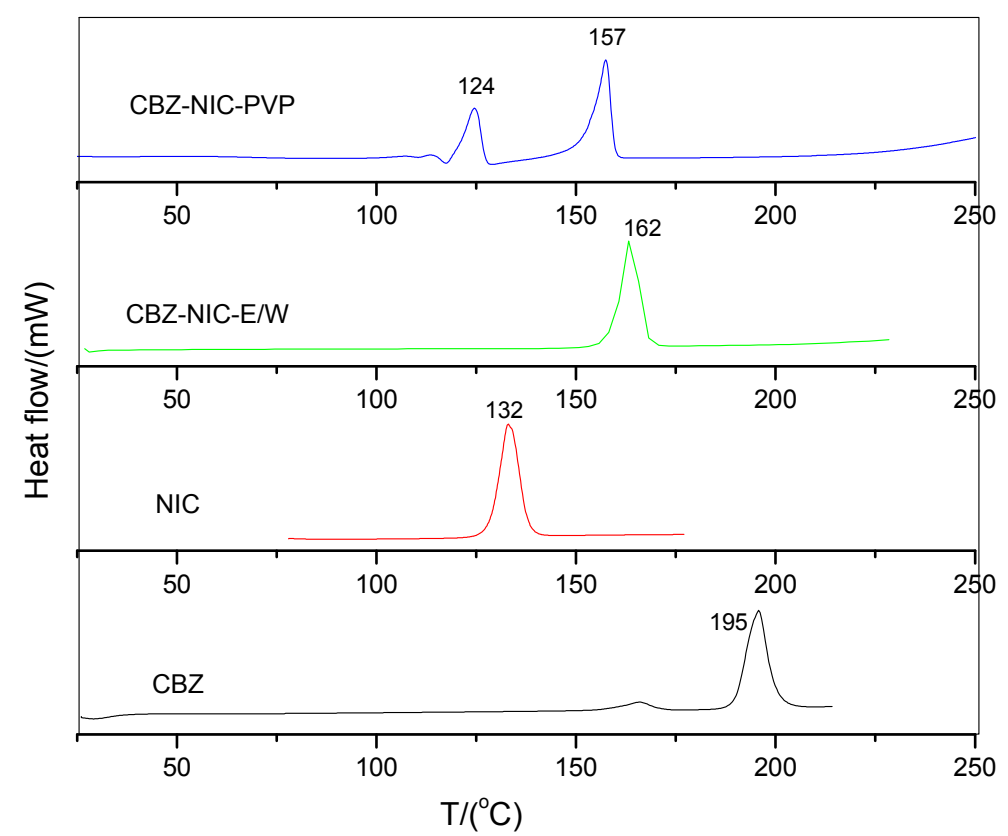

Figure 4. Differential Scanning Calorimetry (DSC) curves of CBZ, NIC, CBZ-NIC-E/W, and CBZ-NIC-PVP.

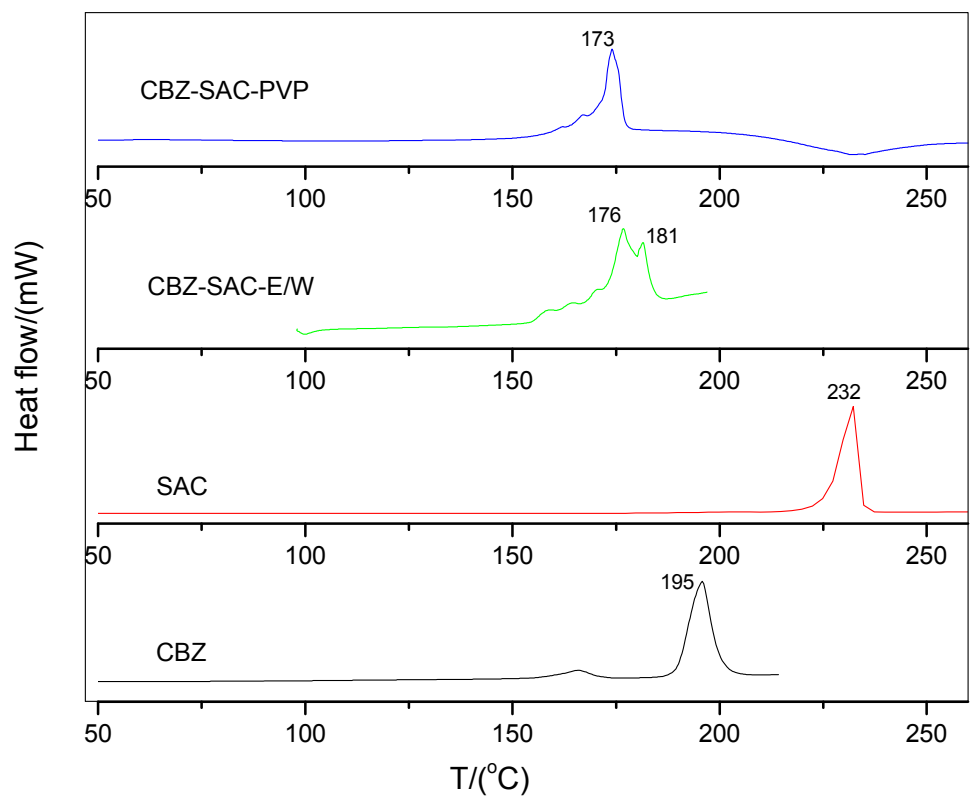

Figure 5. DSC curves of CBZ, SAC, CBZ-SAC-E/W, and CBZ-SAC-PVP.

\subsection{PXRD Analysis}

The PXRD characterization results of CBZ-NIC cocrystal screening products and the original material of CBZ and NIC are shown in Figure 6. The reference data for CBZ-NIC cocrystal characteristic PXRD peaks are also shown in Figure 6. As shown in Figure 6, sample CBZ-NIC-PVP shows both CBZ

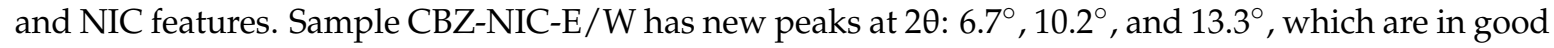
agreement with reported CBZ-NIC characteristic PXRD peaks [14,37]. Base on above characterizations, pure CBZ-NIC cocrystal was successfully prepared in 1:1 volume ratio ethanol-water solvent mixture, and there is no CBZ-NIC cocrystal formed in PVP solution, the product obtained in PVP solution is a mixture. 


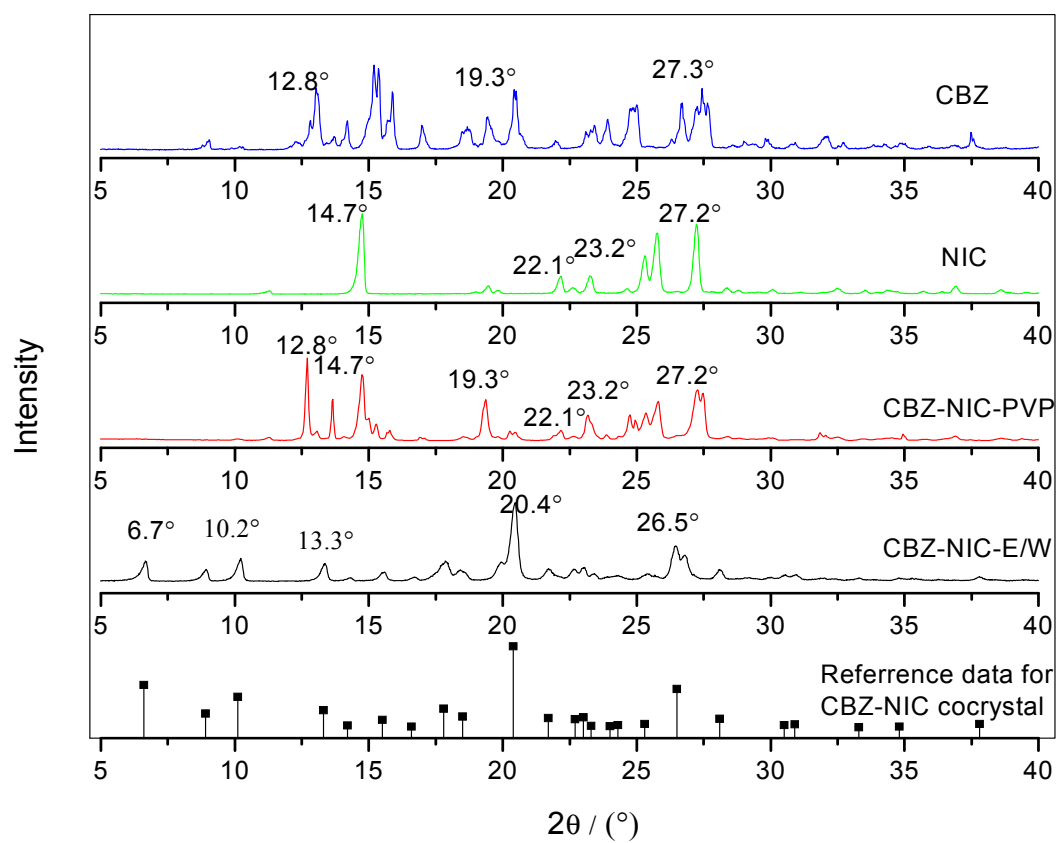

Figure 6. Powder X-ray diffraction (PXRD) spectra of CBZ, NIC, CBZ-NIC-PVP, CBZ-NIC-E/W, and CBZ-NIC-cocrystal.

The PXRD characterization results for CBZ-SAC samples are shown in Figure 7. Sample CBZ-SAC-E/W shows a mixture feature with both CBZ and SAC characteristic peaks. Sample CBZ-NIC-PVP has cocrystal features at $20: 7.0^{\circ}, 9.0^{\circ}, 12.3^{\circ}$, and $14.1^{\circ}$ [12]. In sum, the CBZ-SAC cocrystal screening product prepared in ethanol-water is a mixture of $\mathrm{CBZ}$ and $\mathrm{SAC}$, and the sample prepared in PVP solution is pure CBZ-SAC cocrystal.

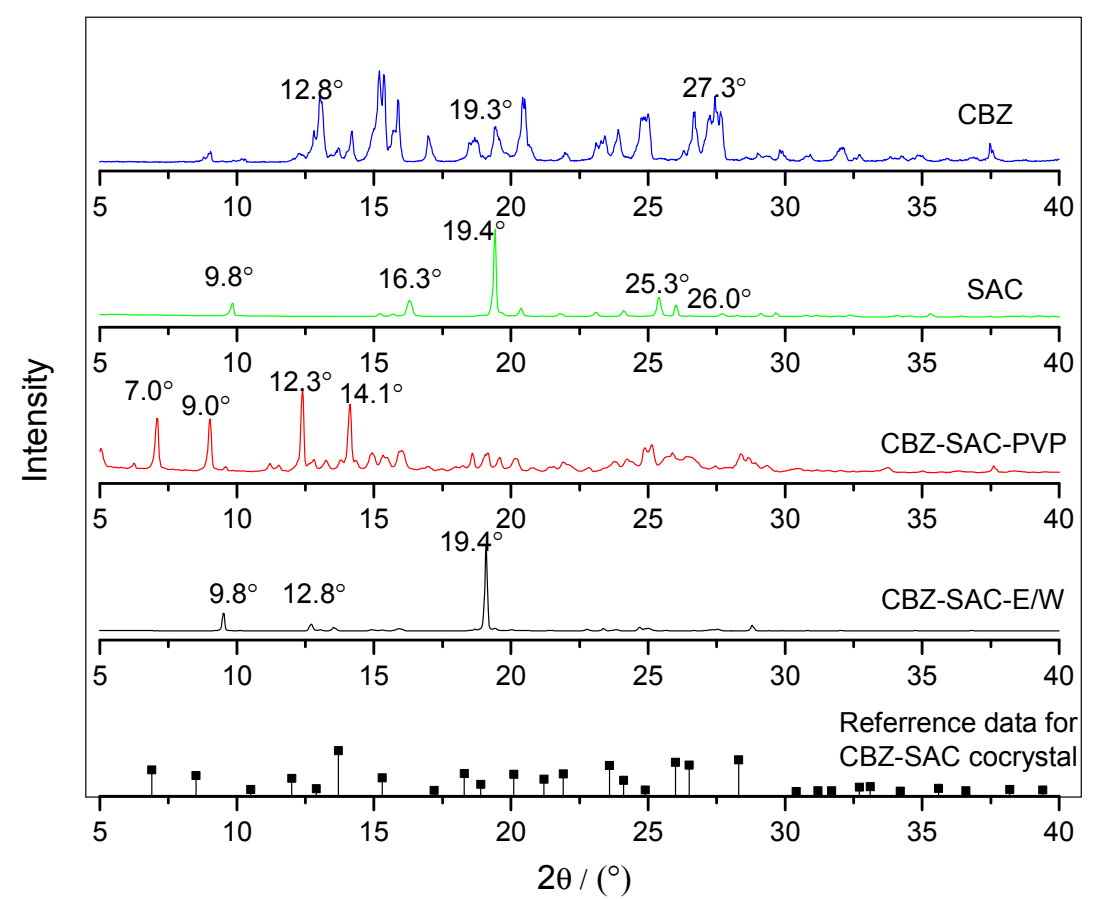

Figure 7. PXRD spectra of CBZ, SAC, CBZ-SAC-E/W, and CBZ-SAC-PVP. 


\subsection{Molecular Structures of CBZ Cocrystals}

The above characterizations demonstrated the formation of CBZ-NIC cocrystal and CBZ-SAC cocrystal. The molecular structures of CBZ cocrystal are shown in Figure 8 [38,39]. CBZ-NIC cocrystal, Figure $8 \mathrm{a}$, has an amide-to-amide structure. It is formed through $\mathrm{N}-\mathrm{H} \ldots \mathrm{O}=\mathrm{C}$ hydrogen bonds in which the carboxamide groups from both CBZ and NIC provide hydrogen bond donors and acceptors. For CBZ-SAC cocrystal, the coformer, SAC, has $\mathrm{S}=\mathrm{O}$ group as hydrogen bond acceptor and N-H group as hydrogen bond donor. In CBZ-SAC cocrystal structure, Figure 8b, SAC is held by S=O ... H-N and $\mathrm{N}-\mathrm{H} \ldots \mathrm{O}=\mathrm{C}$ hydrogen bonds with carboxamide groups of CBZ.

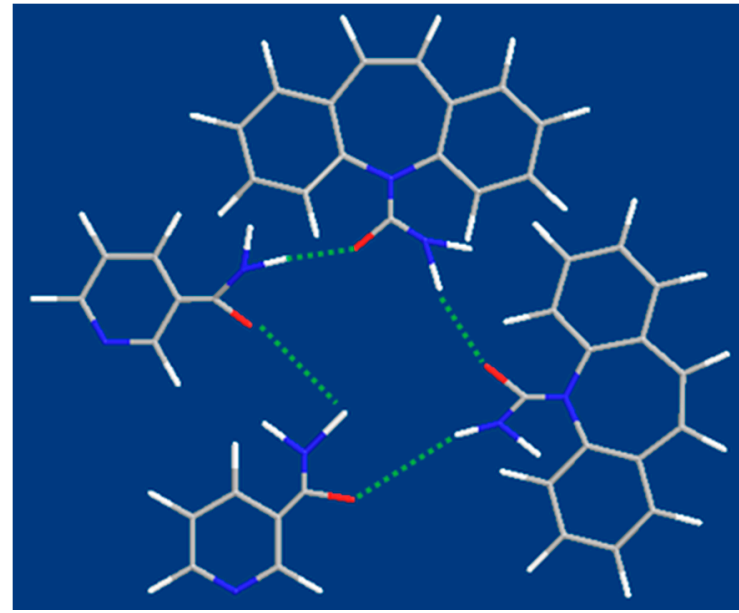

(a)

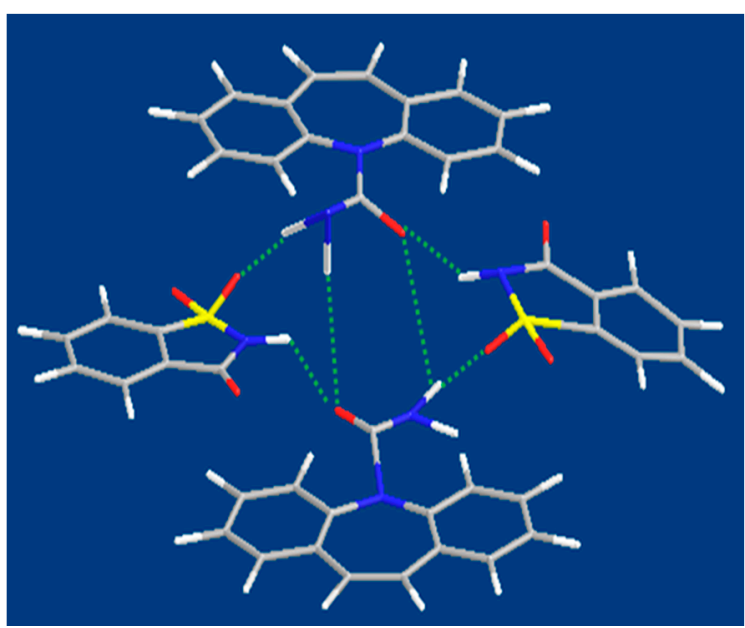

(b)

Figure 8. Illustration of the interaction between CBZ and coformer in cocrystal. (a) CBZ-NIC cocrystal (b) CBZ-SAC cocrsytal.

\subsection{Discussion of the Effects of PVP on CBZ Cocrystal Preparation}

In this study, the effects of PVP on CBZ-NIC and CBZ-SAC cocrystal formation process have been researched. PVP is one of the most researched polymers made from the monomer of $N$-Vinyl-2-pyrrolidone, which has been widely used in the food and pharmacy industries as an additive or excipient [27,30-34]. In the macromolecule structure of PVP, it has both flexible long carbon chain and side ring groups, pyrrolidinone, which offer some rigid property to PVP structure. The flexible chain and the rigid side group together can impart PVP adjustable steric structure properties. PVP is highly soluble in water, ethanol, and other polar solvents. The solubility of PVP in water/ethanol is about $1000 \mathrm{mg} / \mathrm{mL}$. In this study, PVP could remain dissolved state in solution due to its high solubility. PVP has a broad endothermal peak around 50-100 ${ }^{\circ} \mathrm{C}$ [40-42], which is not observed in DSC curves of any cocrystal screening products. Besides, the PXRD spectrum of PVP, shown in Figure 9, has broad peaks around $11^{\circ}$ and $20^{\circ}$ corresponding to the PVP crystalline phase, although PVP has a poor crystallinity degree. While these characteristic broad peaks of PVP have not been found in PXRD spectrum of any samples prepared in PVP solutions. The test results of DSC and PXRD demonstrate that PVP did not precipitate during the cooling and slow evaporation cocrystal preparation process.

For CBZ-NIC cocrystal preparation, CBZ-NIC cocrystal screening product prepared in ethanol-water solvent mixture has been characterized as pure CBZ-NIC cocrystal. This demonstrates that a water-ethanol solvent mixture with 1:1 volume ratio is a promising solvent for CBZ-NIC cocrystal preparation. CBZ is poorly soluble in water, while NIC has a very high solubility in water. The solubility difference between the two cocrystal components can be decreased a lot in an ethanol-water solvent mixture, which facilitates the formation of CBZ-NIC complexation and the consequently precipitation of CBZ-NIC cocrystal. The sample prepared in PVP solution was characterized as a physical mixture, which indicated that the addition of PVP play an inhibition role in CBZ-NIC cocrystal formation. 
The solubility data show that PVP has no adverse effect on components dissolving. Both CBZ and NIC have some increase in their solubility in the PVP solution, but their solubility difference was also increased due to differential solubilizaiton of the two components. The inhibition on cocrystal formation could result from the interaction between the pyrrolidinone ring in PVP and the amide group in NIC. The carbonyl group in pyrrolidinone and the amine group in amide formed a relative strong connection, and this connection more competitive than that between CBZ and NIC. Thus, the complex and precipitation of CBZ-NIC cocrystal was affected by addition of PVP.

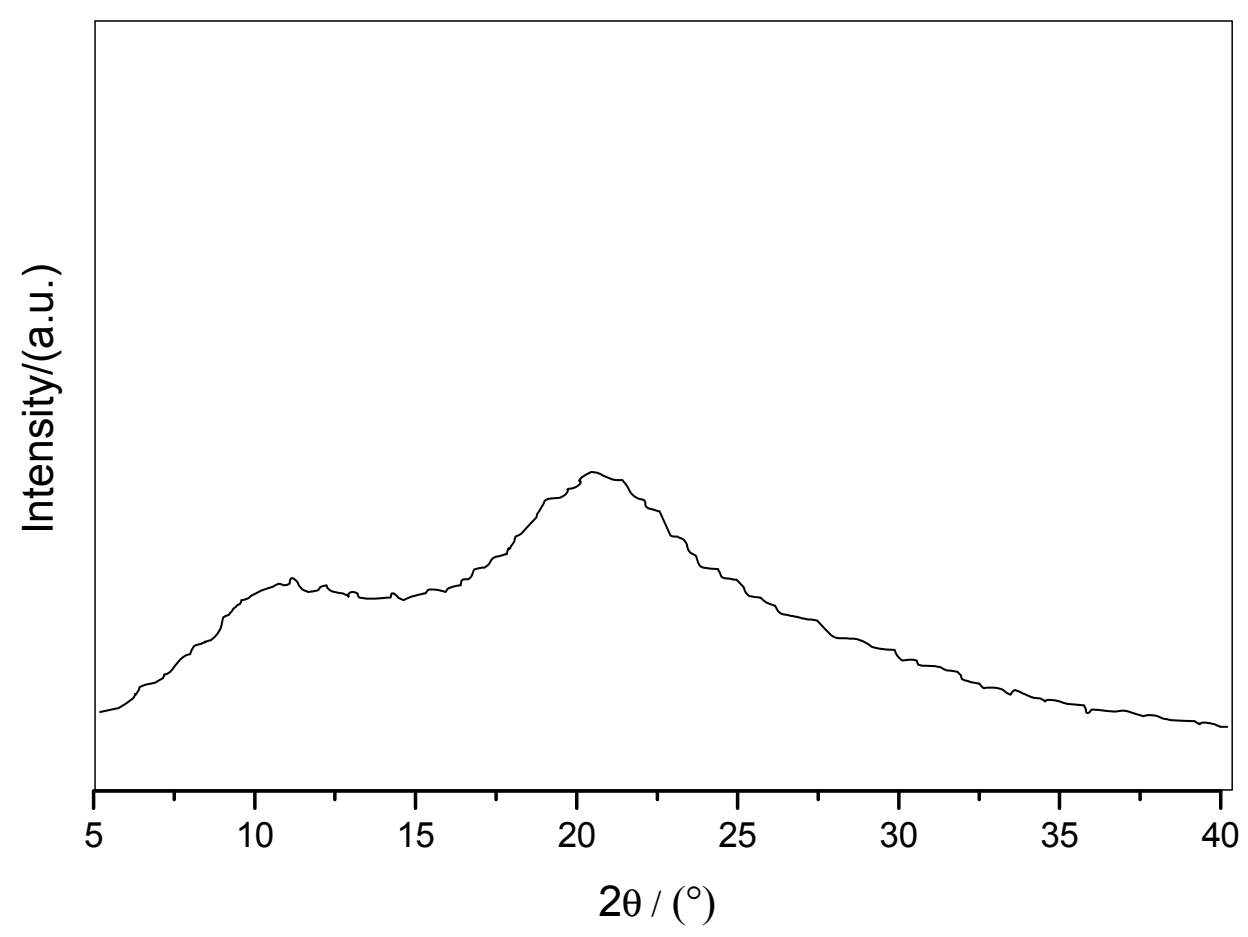

Figure 9. PXRD spectra of PVP.

Although the solubility difference between CBZ and SAC has also been decreased in ethanol-water solvent mixture, the sample prepared in ethanol-water mixture was demonstrated as a physical mixture. In the PVP solution, the solubility difference has been further decreased slightly due to differential solubilization of two components. The sample prepared in the PVP solution was characterized as pure CBZ-SAC cocrystal. The addition of PVP facilitates the formation of CBZ-SAC cocrystal. The effect of PVP on CBZ-SAC cocrystal formation is opposite to that on the CBZ-NIC cocrystal. H-bond connection could also form between PVP and SAC, same as that formed between PVP and NIC. But the double ring structure in SAC molecule may generate larger steric hindrance. PVP may interfere the intermolecular interactions between CBZ and SAC but is not essential to disturb the formation of the CBZ-SAC cocrystal structure [43]. On the contrary, the addition of PVP induced the formation of CBZ-SAC cocrystal. It is assumed that PVP can help the nucleation of CBZ-SAC cocrystal.

\section{Conclusions}

In order to study the effect of PVP on cocrystal formation, we attempted to prepare CBZ-NIC cocrystal and CBZ-SAC cocrystal in a 1:1 volume ratio ethanol-water solvent mixture and polyvinyl pyrrolidone (PVP) solution. Cocrystal screening products were characterized by FTIR, DSC, and PXRD techniques. Characterization results show that in an ethanol-water solvent mixture, pure CBZ-NIC cocrystal can be prepared, while CBZ-SAC cocrystal cannot be formed. The addition of PVP can inhibit CBZ-NIC cocrystal formation and facilitate CBZ-SAC cocrystal formation, which could be the result of integration of the solubility enhancement ability of PVP and the intermolecular 
interaction between PVP and cocrystal components. Polymers are complicated macromolecules; their properties vary with the monomer species, polarity, molecular weight, and distribution, etc. The properties of pharmaceutical cocrystals also vary a lot with different APIs or CCFs. Thus, the conclusion regarding the influences of polymers on pharmaceutical cocrystals formation cannot be perfectly summarized based on limited experiments. Further research is necessary to supplement and improve the conclusions.

Acknowledgments: This study has received support from the Science and Technology Project of Hebei Province (No. 16211505), the Natural Science Foundation of Hebei Province Youth Fund (No. E2016209327), and the Doctoral Research Fund of North China University of Science and Technology (No. 28405399).

Author Contributions: Hao Zhang, Ying Zhu and Ning Qiao designed the experiments; Hao Zhang and Ying Zhu performed the experiments; Yang Chen and Linghuan Gao contributed materials and analysis; Hao Zhang, Ying Zhu and Ning Qiao wrote the paper.

Conflicts of Interest: The authors declare no conflict of interest.

\section{References}

1. Aakeröy, C.B.; Salmon, D.J. Building co-crystals with molecular sense and supramolecular sensibility. Cryst. Eng. Commun. 2005, 7, 439-448. [CrossRef]

2. Qiao, N.; Li, M.; Schlindwein, W.; Malek, N.; Davies, A.; Trappitt, G. Pharmaceutical cocrystals: An overview. Int. J. Pharm. 2011, 419, 1-11. [CrossRef] [PubMed]

3. Vishweshwar, P.; McMahon, J.A.; Bis, J.A.; Zaworotko, M.J. Pharmaceutical co-crystals. J. Pharm. Sci. 2006, 95, 499-516. [CrossRef] [PubMed]

4. Shan, N.; Zaworotko, M.J. The role of cocrystals in pharmaceutical science. Drug Discov. Today 2008, 13, 440-446. [CrossRef] [PubMed]

5. Blagden, N.; De Matas, M.; Gavan, P.T.; York, P. Crystal engineering of active pharmaceutical ingredients to improve solubility and dissolution rates. Adv. Drug Deliv. Rev. 2007, 59, 617-630. [CrossRef] [PubMed]

6. Schultheiss, N.; Newman, A. Pharmaceutical Cocrystals and Their Physicochemical Properties. Cryst. Growth Des. 2009, 9, 2950-2967. [CrossRef] [PubMed]

7. McNamara, D.P.; Childs, S.L.; Giordano, J.; Iarriccio, A.; Cassidy, J.; Shet, M.S.; Mannion, R.; O'donnell, E.; Park, A. Use of a glutaric acid cocrystal to improve oral bioavailability of a low solubility API. Pharm. Res. 2006, 23, 1888-1897. [CrossRef] [PubMed]

8. Good, D.J.; Rodríguez-Hornedo, N.R. Solubility Advantage of Pharmaceutical Cocrystals. Cryst. Growth Des. 2009, 9, 2252-2264. [CrossRef]

9. Qiao, N.; Wang, K.; Schlindwein, W.; Davies, A.; Li, M. In situ monitoring of carbamazepine-nicotinamide cocrystal intrinsic dissolution behaviour. Eur. J. Pharm. Biopharm. 2013, 83, 415-426. [CrossRef] [PubMed]

10. Li, M.; Qiao, N.; Wang, K. Influence of Sodium Lauryl Sulfate and Tween 80 on Carbamazepine-Nicotinamide Cocrystal Solubility and Dissolution Behaviour. Pharmaceutics 2013, 5, 508-524. [CrossRef] [PubMed]

11. Rahman, Z.; Samy, R.; Sayeed, V.A.; Khan, M.A. Physicochemical and mechanical properties of carbamazepine cocrystals with saccharin. Pharm. Dev. Technol. 2012, 17, 457-465. [CrossRef] [PubMed]

12. Porter, W.W., III; Elie, S.C.; Matzger, A.J. Polymorphism in Carbamazepine Cocrystals. Cryst. Growth Des. 2008, 8, 14-16. [CrossRef] [PubMed]

13. Chieng, N.; Hubert, M.; Saville, D.; Rades, T.; Aaltonen, J. Formation Kinetics and Stability of Carbamazepine-Nicotinamide Cocrystals Prepared by Mechanical Activation. Cryst. Growth Des. 2009, 9, 2377-2386. [CrossRef]

14. Seefeldt, K.; Miller, J.; Alvarez-Nunez, F.; Rodriguez-Hornedo, N. Crystallization pathways and kinetics of carbamazepine-nicotinamide cocrystals from the amorphous state by in situ thermomicroscopy, spectroscopy and calorimetry studies. J. Pharm. Sci. 2007, 96, 1147-1158. [CrossRef] [PubMed]

15. Friščić, T.; Jones, W. Recent Advances in Understanding the Mechanism of Cocrystal Formation via Grinding. Cryst. Growth Des. 2009, 9, 1621-1637. [CrossRef]

16. Abd Hashib, S.; Anuar, N.; Jamburi, N.; Ahmad, N.F.; Abd Rahim, S. Screening for Ibuprofen-Sachharin Co-Crystal Formation in Wet Milling. Appl. Mech. Mater. 2015, 754-755, 1002-1006. [CrossRef] 
17. Trask, A.V.; Motherwell, W.D.S.; Jones, W. Solvent-drop grinding: Green polymorph control of cocrystallisation. Chem. Commun. 2004, 890-891. [CrossRef] [PubMed]

18. Wang, I.C.; Lee, M.J.; Sim, S.J.; Kim, W.S.; Chun, N.H.; Choi, G.J. Anti-solvent co-crystallization of carbamazepine and saccharin. Int. J. Pharm. 2013, 450, 311-322. [CrossRef] [PubMed]

19. Nishimaru, M.; Kudo, S.; Takiyama, H. Cocrystal production method reducing deposition risk of undesired single component crystals in anti-solvent cocrystallization. J. Ind. Eng. Chem. 2016, 36, 40-43. [CrossRef]

20. Childs, S.L.; Rodríguez-Hornedo, N.; Reddy, L.S.; Jayasankar, A.; Maheshwari, C.; McCausland, L.; Shipplett, R.; Stahly, B.C. Screening strategies based on solubility and solution composition generate pharmaceutically acceptable cocrystals of carbamazepine. Cryst. Eng. Comm. 2008, 10, 856-864. [CrossRef]

21. Rodríguez-Hornedo, N.; Nehm, S.J.; Seefeldt, K.F.; Pagan-Torres, Y.; Falkiewicz, C.J. Reaction crystallization of pharmaceutical molecular complexes. Mol. Pharm. 2006, 3, 362-367. [CrossRef] [PubMed]

22. Shen, Z.; Xie, C.; Du, W.; Yin, Q. Preparation of Ibuprofen-Nicotinamide Cocrystal and Its Solubility Measurement. Chem. Ind. Eng. 2014, 31, 38-42. [CrossRef]

23. Rager, T.; Hilfiker, R. Cocrystal Formation from Solvent Mixtures. Cryst. Growth Des. 2010, 10, 3237-3241. [CrossRef]

24. Gu, C.H.; Young, V.; Grant, D.J.W. Polymorph screening: Influence of solvents on the rate of solvent-mediated polymorphic transformation. J. Pharm. Sci. 2001, 90, 1878-1890. [CrossRef] [PubMed]

25. Di Profio, G.; Grosso, V.; Caridi, A.; Caliandro, R.; Guagliardi, A.; Chita, G.; Curcio, E.; Drioli, E. Direct production of carbamazepine-saccharin cocrystals from water/ethanol solvent mixtures by membrane-based crystallization technology. Cryst. Eng. Comm. 2011, 13, 5670-5673. [CrossRef]

26. Guo, M.; Wang, K.; Hamill, N.; Lorimer, K.; Li, M. Investigating the Influence of Polymers on Supersaturated Flufenamic Acid Cocrystal Solutions. Mol. Pharm. 2016, 13. [CrossRef] [PubMed]

27. Qiu, S.; Lai, J.; Guo, M.; Wang, K.; Lai, X.; Desai, U.; Juma, N.; Li, M. Role of polymers in solution and tablet based carbamazepine cocrystal formulations. Cryst. Eng. Comm 2016, 18, 2664-2678. [CrossRef]

28. Huang, N.; Rodríguez-Hornedo, N. Engineering cocrystal solubility, stability, and pH(max) by micellar solubilization. J. Pharm. Sci. 2011, 100, 5219-5234. [CrossRef] [PubMed]

29. Gift, A.D.; Luner, P.E.; Luedeman, L.; Taylor, L.S. Influence of polymeric excipients on crystal hydrate formation kinetics in aqueous slurries. J. Pharm. Sci. 2008, 97, 5198-5211. [CrossRef] [PubMed]

30. Higuchi, T.; Kuramoto, R. Study of possible complex formation between macromolecules and certain pharmaceuticals. II. Polyvinylpyrrolidone with p-aminobenzoic acid, aminopyrine, benzoic acid, salicylic acid, p-hydroxybenzoic acid, m-hydroxybenzoic acid, citric acid, and phenobarbit. J. Am. Pharm. Assoc. 1954, 43, 398-401. [CrossRef]

31. Karavas, E.; Ktistis, G.; Xenakis, A.; Georgarakis, E. Effect of hydrogen bonding interactions on the release mechanism of felodipine from nanodispersions with polyvinylpyrrolidone. Eur. J. Pharm. Biopharm. 2006, 63, 103-114. [CrossRef] [PubMed]

32. Van den Mooter, G.; Wuyts, M.; Blaton, N.; Busson, R.; Grobet, P.; Augustijns, P.; Kinget, R. Physical stabilisation of amorphous ketoconazole in solid dispersions with polyvinylpyrrolidone K25. Eur. J. Pharm. Biopharm. 2001, 12, 261-269. [CrossRef]

33. D'Souza, A.J.; Schowen, R.L.; Topp, E.M. Polyvinylpyrrolidone-drug conjugate: Synthesis and release mechanism. J. Control. Release 2004, 94, 91-100. [CrossRef] [PubMed]

34. Gupta, P.; Kakumanu, V.K.; Bansal, A.K. Stability and solubility of celecoxib-PVP amorphous dispersions: A molecular perspective. Pharm. Res. 2004, 21, 1762-1769. [CrossRef] [PubMed]

35. Daimay, L.V. Handbook of Infrared and Raman Characteristic Frequencies of Organic Molecules; Academic Press: Boston, MA, USA, 1991.

36. Li, R.; Mao, H.; Gong, J. Preparation and Characterisation Study of Carbamazpine-Saccharin Cocrystal. 2011. Available online: http:/ / www.paper.edu.cn (accessed on 23 November 2011).

37. Liu, X.; Lu, M.; Guo, Z.; Huang, L.; Feng, X.; Wu, C. Improving the chemical stability of amorphous solid dispersion with cocrystal technique by hot melt extrusion. Pharm. Res. 2012, 29, 806-817. [CrossRef] [PubMed]

38. Berry, D.J.; Seaton, C.C.; Clegg, W.; Harrington, R.W.; Coles, S.J.; Horton, P.N.; Hursthouse, M.B.; Storey, R.; Jones, W.; Friscic, T.; et al. Applying hot-stage microscopy to co-crystal screening: A study of nicotinamide with seven active pharmaceutical ingredients. Cryst. Growth Des. 2008, 8, 1697-1712. [CrossRef] 
39. Fleischman, S.G.; Kuduva, S.S.; McMahon, J.A.; Moulton, B.; Bailey Walsh, R.D.; Rodríguez-Hornedo, N.; Zaworotko, M.J. Crystal Engineering of the Composition of Pharmaceutical Phases: Multiple-Component Crystalline Solids Involving Carbamazepine. Cryst. Growth Des. 2003, 3, 909-919. [CrossRef]

40. Zerlia, T.; Marini, A.; Berbenni, V.; Massarotti, V.; Giordano, F.; La Manna, A.; Bettinetti, G.P.; Margheritis, C. Solid State Interaction study on the system polyvinylpyrrolidone-XL/trimethoprim. Solid State Ion. 1989, 32, 613-624. [CrossRef]

41. Lin, S.P.; Hou, Y.C.; Liao, T.Y.; Tsai, S.Y. Enhancing the bioavailability of magnolol in rabbits using melting solid dispersion with polyvinylpyrrolidone. Drug Dev. Ind. Pharm. 2014, 40, 330-337. [CrossRef] [PubMed]

42. Guo, S.R.; Guo, L. Effects of PVP K30 on Aqueous Solubility and Dissolution Properti of Daidzein. J. Chin. Pharm. Sci. 2004, 13, 42-48. [CrossRef]

43. Shete, A.; Murthy, S.; Thorat, B.; Yadav, A.; Sajane, S.; Sakhare, S.; Doijad, R. Studies on effect of hydrophilic polymers on physicochemical properties of itraconazole cocrystals. Future J. Pharm. Sci. 2017. [CrossRef]

2017 by the authors. Licensee MDPI, Basel, Switzerland. This article is an open access article distributed under the terms and conditions of the Creative Commons Attribution (CC BY) license (http:// creativecommons.org/licenses/by/4.0/). 